\title{
Repairing inappropriately specified utterances: Revision or restart?
}

\author{
HELEEN T. BOLAND \\ University of Utrecht, Utrecht, The Netherlands \\ ROBERT J. HARTSUIKER \\ Ghent University, Ghent, Belgium \\ MARTIN J. PICKERING \\ University of Edinburgh, Edinburgh, Scotland \\ and \\ ALBERT POSTMA \\ University of Utrecht, Utrecht, The Netherlands
}

\begin{abstract}
In two experiments, we investigated repair of inappropriately specified utterances (e.g., blue square instead of dark blue square) to determine whether speakers can simply revise a speech plan or must begin anew. In Experiment 1, speakers produced a prime utterance followed by a target utterance that differed from the prime in only one word, but in Experiment 2, they only planned the prime but produced the target. If speakers must repair by restarting, both situations would involve planning from scratch, with a possible benefit of residual activation. If they can repair by revising, though, only the first situation would involve planning from scratch, and the second situation would involve revision of a plan. The targets had either one word more (addition) or fewer (deletion) than the prime sentences. The restart hypothesis predicted that any cost of addition over deletion should be similar in the two experiments. In contrast, the revision hypothesis predicted an extra cost of addition in Experiment 2, because addition involves retrieval of an extra word. In support of the revision hypothesis, there was no difference between additions and deletions in Experiment 1, but in Experiment 2 additions took longer than deletions. We conclude that speakers can repair utterances by revising a speech plan.
\end{abstract}

Sometimes people produce utterances smoothly, without making any changes. But at other times they are just getting ready to produce an utterance when they change their minds. Although there has been some research into the nature of such repairs (e.g., Berg, 1986; Blackmer \& Mitton, 1991; Levelt, 1983), and although there are theories about the way in which people monitor their own planned utterances (see Postma, 2000), little is known about the mechanisms that underlie repairs. In particular, we do not know whether people can revise their plans or whether they have to restart from scratch.

Postma and Kolk (1993) suggested that if speakers detect a problem in their speech plan as a result of selfmonitoring, they can simply restart. Suppose a speaker has planned to say "blue square" but changes his or her

This research was sponsored by Economic and Social Research Council (U.K.) Grant R000223812 awarded to R.J.H. and M.J.P. and a grant from the Netherlands Organization for Fundamental Research (NWO 440-20-000) awarded to A.P. We thank Zenzi Griffin and two anonymous reviewers for helpful comments on an earlier version of the manuscript. Correspondence relating to this article may be sent to R. J. Hartsuiker, Department of Experimental Psychology, Ghent University, Henri Dunantlaan 2, 9000 Ghent, Belgium (e-mail: robert.hartsuiker (a) ugent.be). mind and produces "dark blue square" (because the former is not sufficiently discriminatory). Although he or she plans the utterance again from scratch, residual activation of previously selected representations (such as those for blue and square, and perhaps syntactic information associated with noun phrases) may facilitate the restart (Hartsuiker \& Kolk, 2001). However, it is also possible that representations are briefly inhibited after selection, perhaps to prevent repeated selection or to prevent initially incorrect representations from being selected again (Dell, Burger, \& Svec, 1997; MacKay, 1987). Since MacKay also proposed a rebound of activation after inhibition, any pattern of inhibition or facilitation may depend on the precise time course of production.

In contrast, the revision hypothesis assumes that speakers have access to stored plans and revise these plans as required (van Wijk \& Kempen, 1987). A speaker would then not need to plan entirely from scratch, but would use aspects of the initially planned representations that were still appropriate (e.g., a syntactic frame with the words inserted in the appropriate positions). Thus, he or she might produce "dark blue square" using the plan for blue square and adding the word dark. Whereas the restart hypothesis has the advantage of simplicity (there is no need to 
consider the specific ways in which the utterance was problematic), the revision hypothesis has the advantage of efficiency (the speaker does not have to reselect representations that were initially correct).

We contrasted these two hypotheses by considering the time course of noun phrase production when production of a target noun phrase was preceded by the actual production of either the same noun phrase or one differing in only one word (Experiment 1) or when the first noun phrase was planned but not produced (Experiment 2). Specifically, we compared the production of target phrases that contained a word fewer than the prime (e.g., dark blue square $\rightarrow$ blue square) with target phrases that contained a word more (e.g., blue square $\rightarrow$ dark blue square).

Consider the situation in which the speaker overtly produces the prime. For both targets with a word fewer and with a word more, there is considerable overlap between prime and target (the structure is a noun phrase, and the conceptual, lexical, and phonological representations of blue and square are shared), and there are also differences (the representations of dark are not shared, and the internal structure of the noun phrase is different). Priming might well occur under such conditions, because the production of the prime might facilitate production of the target, most obviously at the level of lexical repetition (e.g., Wheeldon \& Monsell, 1992). If so, we might expect more priming when deleting than when adding a word, because adding introduces a new word that has not been primed. However, it is also possible that the effects of adding versus removing one word (i.e., the words light and dark, both of which are used repeatedly in the experiments) are negligible. Experiment 1 tested these propositions.

Now consider the situation in which the speaker only plans the prime and then produces the target. According to the restart hypothesis, this situation is analogous to just having said the prime, and the production of the target will therefore be primed in a similar fashion as in Experiment 1 . According to the revision hypothesis, however, the speaker will revise a stored representation. For both additions and deletions, this revision will require both a change at the message level and adjustment of the syntactic frame, but additions will also require the retrieval of an additional word from the lexicon (e.g., dark) and its insertion in the syntactic frame. Therefore, the revision hypothesis strongly suggests that additions will take longer to process than deletions and that such a difference will be larger than any difference due to priming only.

In our experiments, we encouraged participants to produce or prepare a picture description and then presented another picture that occasionally required a different description (cf. van Wijk \& Kempen, 1987). The pictures showed two simple, colored geometrical objects, and participants prepared or produced a contextually appropriate name for the one that was underlined (the selected object). After an interval that allowed ample time to describe the first picture, another picture ap- peared that showed the same selected object. On $10 \%$ of the trials, however, the unselected object changed, so the initial description was no longer applicable (it was either too specific or not specific enough). ${ }^{1}$ Therefore, we expected speakers to either produce a different description for the target picture (Experiment 1) or repair their initial plan (Experiment 2).

To bring about additions and deletions to the planned utterance, we manipulated whether the color of an object was light or dark (its shade). Two objects of the same shape and color but different shades can be distinguished by mentioning the shade (e.g., dark blue square), but if the objects have different colors, there is no need to mention shade (e.g., blue square is sufficient if the other square is green). Thus, we manipulated whether the unselected object differed in color or just shade from the selected object. In critical trials, the unselected object changed in ways that were intended to elicit either addition or deletion repairs. In Experiment 1, participants provided a description of both the prime and target. In Experiment 2, they planned the prime but did not produce it. Experiment 1 therefore tested the restart situation (with a potential benefit from residual activation), and Experiment 2 tested for the presence of repair processes beyond restart and residual activation.

\section{EXPERIMENT 1}

\section{Method}

Participants. Twenty-four University of Edinburgh students (18 females, 6 males) participated in exchange for $£ 3$. They were all native English speakers.

Materials. We used 160 pairs of pictures. Each picture showed two objects, both of the same shape (moon, heart, star, sun, square, circle, triangle, oval, cross, or arrow). Each shape was one of three colors (blue, green, or brown) and one of three shades (light, medium, or dark). In each picture, one object was underlined. For each pair, one picture was the prime and one was the target. The two objects in each prime and corresponding target phase always had the same shape but were either the same color or different colors. The selected object was always a medium shade, but the unselected object was either a dark or light shade of the same color (requiring a description of the selected object that mentioned the shade) or a medium shade of a different color (requiring a description of the selected object that did not mention the shade).

Of the 160 prime-target pairs, 32 were experimental items and 128 were filler items. Each of the 32 target items occurred in four conditions obtained by crossing the factors of compatibility (objects in prime and target phases identical or different) and shade (shade information required or not required to distinguish the target); see Figure 1.

In the compatible, target with shade condition, the objects in the prime and target phases were the same. In addition, the two objects were the same color and the unselected object was either light or dark, so an appropriate description of the selected object included the shade. In the compatible, target without shade condition, the objects in the prime and target phases were also the same, but the two objects were different colors of medium shade, so an appropriate description would not include the shade. In the incompatible, target with shade (or addition) condition, the unselected object changed: It was a different color (of medium shade) from the selected object in the prime phase, but a dark or light shade of the same color in the target phase. Therefore, a change to the descrip- 


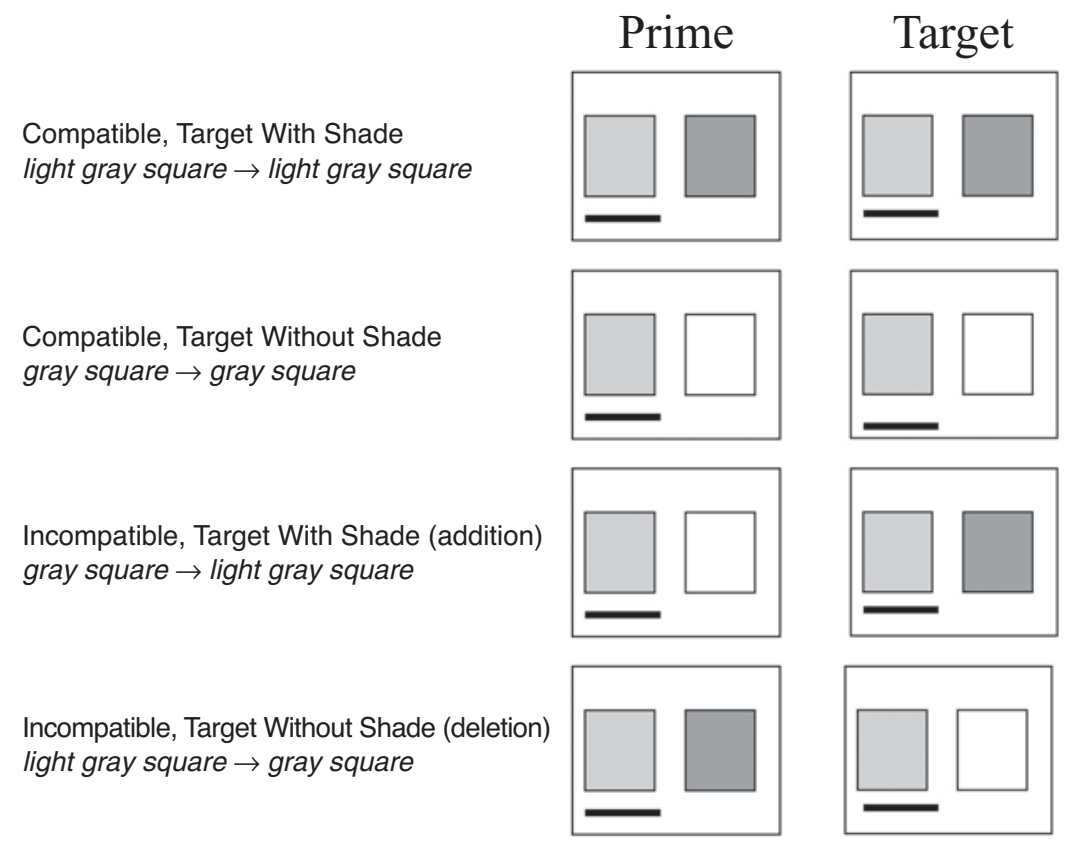

Figure 1. Example trials for all four conditions. Note that the experimental stimuli were presented in color; gray tones are used here only for illustration in black and white.

tion of the prime required adding a word. In the incompatible, target without shade (or deletion) condition, the unselected object also changed: It was a dark or light shade of the same color as the selected object in the prime phase but a different color (of medium shade) in the target phase. Therefore, a change to the description of the prime required deleting a word.

The selected object appeared on the left on half of the experimental trials (balanced across conditions) and on half of the filler trials, and the same object was underlined for both the prime and target phases. The underlined object appeared at the same position in prime and target. We created four lists with the same pseudorandom order, so that each list contained eight items from each condition and exactly one version of every item.

Each trial consisted of a fixation point for $1,000 \mathrm{msec}$, the prime for 2,000 msec, another fixation point for $1,000 \mathrm{msec}$, and the target for $2,000 \mathrm{msec}$. The fixation point for the next trial appeared at the offset of the target. Thus, a given prime-target pair was not temporally separated from the next pair. All pairs were presented at the center of the screen.

The participants were instructed (both orally and in writing) to name the underlined objects in such a way that another person, who would see the same two objects on the screen without underlining, could select the described object. The instruction emphasized that terms like left and right should be avoided. They were informed that subsequent pictures were usually, but not always, identical. The instructions included an example of one picture and a familiarization procedure for the 10 shapes and the three colors (but not for the shades): If the participants named the shapes and colors differently than intended, they were corrected.

During a practice session of 10 trials, the participants were corrected if they used color names other than brown, green, or blue, or if they underspecified the object. The session lasted about $30 \mathrm{~min}$, and the participants could pause halfway through.

Apparatus. The experiment was implemented in E-Prime Version 1.0 (Psychology Software Tools, 2000). Speech was recorded on DAT tape via a high-quality directional microphone. Utterance onset latencies for both prime and target were manually determined using the phonetic software package Praat (Boersma \& Weenink, 1992). The experimenter was blind with respect to whether the targets occurred in the compatible or incompatible conditions.

Scoring and data analysis. One participant was excluded because of software failure, and 2 others were excluded because they produced too many inappropriate descriptions (more than five of the eight trials in at least one condition). A final participant was excluded randomly, so that equal numbers of participants were tested for each list (20 in total). The remaining responses were categorized into correct descriptions (primes, 93.3\%; targets, 86.7\%), overt repairs (e.g., "blue, um, light blue square"; primes, 1.6\%; targets, $3.6 \%$ ), overspecifications (primes, $0.5 \%$; targets, $6.3 \%$ ), underspecifications (primes, $0.9 \%$; targets, $1.7 \%$ ), and speech errors (e.g., wrong color or shape; primes, $3.1 \%$; targets, $0.8 \%$ ). The last four categories were collapsed as errors. Reaction time analyses were conducted on trials in which both prime and target were correct; we excluded a small number of trials for which no latency data were available (primes, $0.6 \%$; targets, $0.9 \%$ ), as well as trials in which the reaction times were more than three standard deviations from the mean (separately for each condition; primes, $0.9 \%$; targets, $0.8 \%$ ). The reaction time data are based on the remaining 513 observations $(80.2 \%)$.

We conducted $2 \times 2$ analyses of variance on target onset latencies and on error proportions, ${ }^{2}$ with compatibility and shade as within-participants and -items factors. Separate analyses were conducted with participants $\left(F_{1}\right)$ and items $\left(F_{2}\right)$ as random variables. The effect of shade on latencies and error frequencies in the primes was assessed with paired $t$ tests.

\section{Results and Discussion}

Mean utterance onset latencies are reported in Table 1. Utterance onset latency of the target was much slower in the incompatible than in the compatible condition, and this effect was highly significant $\left[F_{1}(1,19)=336.16\right.$, 
Table 1

Mean Utterance Onset Latencies ( $M$, in Milliseconds), Standard Deviations $(S D)$, and Percentage Errors $(\% E)$ for Prime and Target by Compatibility and Shade in Experiment 1

\begin{tabular}{lccccccc}
\hline & \multicolumn{3}{c}{ With Shade } & & \multicolumn{3}{c}{ Without Shade } \\
\cline { 2 - 4 } \cline { 6 - 8 } \multicolumn{1}{c}{ Utterance } & $M$ & $S D$ & $\% \mathrm{E}$ & & $M$ & $S D$ & $\% \mathrm{E}$ \\
\hline Prime & 976 & 110 & 7.8 & & 955 & 124 & 4.4 \\
Target & & & & & & \\
$\quad$ Incompatible & 902 & 891 & 7.5 & & 912 & 952 & 8.8 \\
$\quad$ Compatible & 604 & 104 & 1.3 & & 582 & 68 & 1.9 \\
\hline
\end{tabular}

Note-Data from analysis by participants.

$\left.p<.001 ; F_{2}(1,31)=523.58, p<.001\right]$. There was no effect of shade $[F \mathrm{~s}<1]$ and no interaction $\left[F_{1}(1,19)=\right.$ $\left.1.40, p=.25 ; F_{2}<1\right]$. Planned comparisons showed that the two incompatible conditions did not differ [ $t \mathrm{~s}<1]$, and neither did the two compatible conditions $\left[t_{1}(19)=\right.$ $\left.1.06, p=.30 ; t_{2}(31)=1.20, p=.24\right]$. The difference in onset latencies between primes with and without shade was also not significant $\left[t_{1}<1 ; t_{2}=1.03, p=.31\right]$. Finally, compatible targets were produced significantly faster than primes $\left[F_{1}(1,19)=289.48, p<.001 ; F_{2}(1,31)=\right.$ $1,397.1, p<.001]$.

There were marginally more errors for primes with rather than without shade $\left[t_{1}(19)=1.99, p=.06 ; t_{2}(31)=\right.$ $1.94, p=.06]$. There were more errors for incompatible than for compatible targets $\left[F_{1}(1,19)=82.31, p<.001\right.$; $\left.F_{2}(1,31)=63.87, p<.001\right]$ and for targets without shade than for targets with shade $\left[F_{1}(1,19)=5.82, p<\right.$ $\left..05 ; F_{2}(1,31)=5.02, p<.05\right]$. However, these factors interacted significantly $\left[F_{1}(1,19)=5.90, p<.05 ; F_{2}(1,31)=\right.$ $4.38, p<.05]$, and planned comparisons revealed that the effect of shade occurred for the incompatible conditions [i.e., addition vs. deletion; $t_{1}(19)=-2.49 ; p<$ $\left..05 ; t_{2}(31)=-2.25, p<.05\right]$ but not for the compatible conditions $(t \mathrm{~s}<1)$. Because there were more errors in the deletion condition than in the addition condition, benefits of priming did not emerge in the accuracy data (so there was no speed-accuracy tradeoff). This difference was mostly due to inappropriate descriptions: Participants more often failed to delete the shade $(23.1 \%$ of responses in that condition) than to add the shade $(6.9 \%$ of the responses).

Overall, the results demonstrate that residual activation from the prime does not facilitate the production of targets with a word added more than it does production of targets with a word deleted. In fact, the error analysis suggests that production of targets with a word deleted is more difficult than production of targets with a word added. The latter finding may be a result of participants failing to revise overspecifications, since overspecifications (as opposed to underspecifications) do uniquely specify a referent. Importantly, the comparison of primes with identical targets showed a massive priming effect, confirming the assumption that priming occurs in this time frame.

Experiment 2 differed from Experiment 1 in only one respect: Participants planned but did not produce the prime. The restart hypothesis predicted the same result as in Experiment 1 (no difference between the incompatible targets with and without shade). The revision hypothesis, on the other hand, predicted that targets with shade would be more difficult to produce.

\section{EXPERIMENT 2}

\section{Method}

Participants. Thirty-three new University of Edinburgh students ( 26 females, 7 males) participated in exchange for $£ 3$. All were native English speakers.

Materials, Procedure, and Apparatus. These were all identical to those in Experiment 1, except that the participants were instructed to name only the underlined object of each second presentation, but also to prepare the name during the first presentation, so they could say it as quickly as possible on the second presentation.

Scoring and data analysis. We removed 5 participants because of apparatus failures and 8 because they produced too many inappropriate responses (as in Experiment 1). The results are based on the remaining 20 participants ( 5 per list).

As in Experiment 1, responses were divided into correct descriptions $(85.0 \%)$, overt repairs $(2.8 \%)$, overspecifications $(5.8 \%)$, underspecifications (4.4\%), and speech errors (1.6\%). The last four categories were collapsed as errors. No reaction time data were available for $0.5 \%$ of responses. Errors were excluded from reaction time analyses, and so were observations with latencies below $200 \mathrm{msec}$ or more than three standard deviations away from the mean for each condition (3.1\%). The results below are based on the 524 remaining observations $(82 \%)$.

\section{Results and Discussion}

Mean utterance onset latencies are reported in Table 2. As in Experiment 1, onset latencies were much longer for incompatible than for compatible targets $\left[F_{1}(1,19)=\right.$ $\left.184.96, p<.001 ; F_{2}(1,31)=324.68, p<.001\right]$, and there was no significant effect of shade $\left[F_{1}(1,19)=\right.$ $\left.1.64, p=.22 ; F_{2}(1,31)=3.82, p=.06\right]$. More critically, the interaction was significant $\left[F_{1}(1,19)=10.65, p<\right.$ $\left..01 ; F_{2}(1,31)=12.04, p<.01\right]$ : Incompatible targets with shade were produced significantly more slowly than incompatible targets without shade $\left[t_{1}(19)=2.70\right.$, $\left.p<.05 ; t_{2}(31)=3.38, p<.01\right]$. Thus, addition repairs took longer to process than deletion repairs. In the compatible conditions, targets with shade were produced marginally faster than short responses $\left[t_{1}(19)=-2.37\right.$, $\left.p<.05 ; t_{2}(31)=-1.47, p=.15\right]$.

There were more errors for incompatible than for compatible targets $\left[F_{1}(1,19)=21.01, p<.001 ; F_{2}(1,31)=\right.$ $35.86, p<.001]$, but there was no effect of shade $\left[F_{\mathrm{S}}<1\right]$

Table 2

Mean Utterance Onset Latencies ( $M$, in Milliseconds), Standard Deviations $(S D)$, and Percentage Errors $(\% E)$ for Targets With and Without Shade in the Compatible and Incompatible Conditions of Experiment 2

\begin{tabular}{lrrrrrrr}
\hline \multirow{2}{*}{ Target } & \multicolumn{3}{c}{ With Shade } & & \multicolumn{3}{c}{ Without Shade } \\
\cline { 2 - 4 } \cline { 6 - 8 } & $M$ & $S D$ & $\% \mathrm{E}$ & & $M$ & $S D$ & $\% \mathrm{E}$ \\
\hline Incompatible & 971 & 156 & 23.1 & & 876 & 138 & 25.6 \\
Compatible & 556 & 105 & 7.5 & & 598 & 133 & 2.5 \\
\hline
\end{tabular}

Note-Data from analysis by participants. 
and no interaction $\left[F_{1}(1,19)=2.50, p=.13 ; F_{2}(1,31)=\right.$ $1.85, p=.18]$. There was no effect of shade for the incompatible targets $[t \mathrm{~s}<1]$, but in the compatible conditions, there were significantly more errors for responses with than without shade $\left[t_{1}(19)=2.37, p<.05 ; t_{2}(31)=\right.$ $2.27, p<.05]$. The latter finding suggests a speedaccuracy tradeoff in the compatible conditions, which explains the slightly faster reaction times for responses with shade in that condition. Importantly, there was no indication of such a tradeoff in the incompatible conditions.

These results confirm the prediction of the revision hypothesis. It took nearly $100 \mathrm{msec}$ longer to repair an utterance by adding a word than it took to repair by deleting one. The results do not appear to be due to differential priming effects, since Experiment 1 found no difference between the two incompatible target conditions.

\section{Comparison of Experiments 1 and 2}

To determine whether planning versus uttering the prime sentence truly had differential effects for addition versus deletion, further analyses included experiment (1 vs. 2) as a between-participants and a within-items factor, revealing a three-way interaction $\left[F_{1}(1,38)=11.30\right.$, $\left.p<.01 ; F_{2}(1,31)=20.81, p<.001\right]$. A planned comparison of experiment and shade for the incompatible conditions was significant $\left[F_{1}(1,38)=6.37, p<.05\right.$; $\left.F_{2}(1,31)=12.10, p<.01\right]$. Repairing by adding took longer than repairing by deleting, but saying the phrase with shade after saying a prime without shade took as long as the converse condition.

\section{GENERAL DISCUSSION}

In Experiment 1, participants described prime and target pictures; in Experiment 2, they planned a description of the prime and described the target. On critical (incompatible) trials, the appropriate description of the prime differed from that of the target. Experiment 1 revealed no difference in the time to produce an incompatible target with a word more than the prime versus an incompatible target with a word fewer than the prime. In contrast, Experiment 2 showed that repairing the plan for the prime by adding a word took longer than repairing by deleting a word.

We have assumed that participants in Experiment 1 were not considering their target utterances as revisions of their prime utterances, since the prime and target were presented to them as individual pictures to be named. However, they were informed that two successive trials involved similar or identical objects. If participants therefore considered target responses to be repairs, this would not alter the conclusion that repair of a plan involves revision, but it would additionally suggest that overt repair (of something already spoken) does not involve revision. Interestingly, Nooteboom (2005) recently suggested that covert and overt repair are indeed different. According to his interpretation of Dutch speech error corpus data, covert repairing affects errors in forming existing words less often than errors in forming nonwords, but overt repairing affects both types of errors equally.

We now briefly discuss an alternative interpretation that maintains the restart hypothesis. The difference between the two experiments could result from different loci of priming: In prior production, priming could result from repeating the message (because speakers would quickly forget the exact wording of the prime), but in planning without production, priming could result from repeating (sub)lexical representations (because speakers would rehearse the planned words). Therefore, only in Experiment 2 would there be a benefit (in the case of deletions) from lexical priming of all words in the target. However, the suggestion that longer term production-toproduction priming effects are due to residual activation at the message level is incompatible with studies that show phonological priming in tasks requiring overt production of both prime and target and with interstimulus intervals comparable to ours (e.g., Sullivan \& Riffel, 1999; Wheeldon, 2003). Because it is implausible that phonological priming results from repeating aspects of the message, this alternative seems very unlikely.

Two further observations are compatible with the revision hypothesis. First, in spontaneous error repairs the grammatical form of the original utterance constrains the form of the repair (Levelt, 1983; van Wijk \& Kempen, 1987), so a repair is well formed if the concatenation of the original utterance, a completion of the interrupted constituent, a conjunction, and the repair results in a correct sentence. This observation suggests that speakers retain the original utterance and add the repair onto it. Second, some overt repairs refer back to the original utterance with a pronoun, as in "Take the oranges to Elmira, um, I mean take them to Corning" (Core \& Schubert, 1999; see also van Wijk \& Kempen, 1987). Assuming that anaphoric reference requires inspection of the antecedent noun (e.g., so that the pronoun gets the correct grammatical gender), part of the original utterance (oranges in the example) appears to be retained.

To conclude, our results confirm the prediction of the revision hypothesis. Although it is highly likely that priming occurs in the incompatible conditions of both Experiments 1 and 2, the difference in time course between addition and deletion repairs in Experiment 2 cannot be explained by priming. Hence, people do not repair simply by restarting and profiting from residual activation. Instead, repair involves revision of a stored plan.

\section{REFERENCES}

Berg, T. (1986). The aftermath of error occurrence: Psycholinguistic evidence from cut-offs. Language \& Communication, 6, 195-213. Blackmer, E. R., \& MitTon, J. L. (1991). Theories of monitoring and the timing of repairs in spontaneous speech. Cognition, 39, 173-194.

Boersma, P., \& WeEnink, D. (1992). Praat (Version 4.0.34) [Computer software]. Amsterdam: Institute of Phonetic Sciences. Available at http://www.praat.org.

Brennan, S. E., \& Clark, H. H. (1996). Conceptual pacts and lexical choice in conversation. Journal of Experimental Psychology: Learning, Memory, \& Cognition, 22, 1482-1493. 
Core, M. G., \& Schubert, L. K. (1999, July). Speech repairs: A parsing perspective. Paper presented at a satellite meeting of the 14th International Congress of Phonetic Sciences (ICPhS 99), Berkeley, CA.

Dell, G. S., Burger, L. K., \& Svec, W. R. (1997). Language production and serial order: A functional analysis and model. Psychological Review, 104, 123-147.

HARTSUiKer, R. J., \& KolK, H. H. J. (2001). Error monitoring in speech production: A computational test of the perceptual loop theory. Cognitive Psychology, 42, 113-157.

Levelt, W. J. M. (1983). Monitoring and self-repair in speech. Cognition, 14, 41-104.

MacKay, D. G. (1987). The organization of perception and action: A theory for language and other cognitive skills. New York: Springer-Verlag.

Noотевоом, S. G. (2005). Listening to oneself: Monitoring speech production. In R. J. Hartsuiker, R. Bastiaanse, A. Postma, \& F. Wijnen (Eds.), Phonological encoding and monitoring in normal and pathological speech (pp. 167-186). Hove, U.K.: Psychology Press.

Postma, A. (2000). Detection of errors during speech production: A review of speech monitoring models. Cognition, 77, 97-131.

Postma, A., \& Kolk, H. H. J. (1993). The covert repair hypothesis: Prearticulatory repair processes in normal and stuttered disfluencies. Journal of Speech \& Hearing Research, 36, 472-487.

Psychology Software Tools (2000). E-Prime (Version 1.0) [Computer software]. Pittsburgh, PA: Author.

Sullivan, M. P., \& Riffel, B. (1999). The nature of phonological encoding during spoken word retrieval. Language \& Cognitive Processes, 14, 15-45.
VAN WiJK, C., \& KemPen, G. (1987). A dual system for producing selfrepairs in spontaneous speech: Evidence from experimentally elicited corrections. Cognitive Psychology, 19, 403-440.

WHEELDON, L. R. (2003). Inhibitory form priming of spoken word production. Language \& Cognitive Processes, 18, 81-109.

WheELdon, L. R., \& Monsell, S. (1992). The locus of repetition priming of spoken word production. Quarterly Journal of Experimental Psychology, 44A, 723-761.

\section{NOTES}

1. Such repairs are generally regarded as appropriateness rather than error repairs (Levelt, 1983). Underspecifications do not give enough information for the listener to determine what they refer to, but they are not really erroneous. Overspecifications are even less problematic: They merely give more information than necessary, and under some circumstances they are regularly produced (Brennan \& Clark, 1996). For our purposes, it is unimportant whether participants repair because the initial plan is erroneous or simply inappropriate.

2 . In both experiments, analyses on arcsine-transformed error proportions yielded identical conclusions.

(Manuscript received August 28, 2003;

revision accepted for publication September 2, 2004.) 